\title{
AUTHOR INDEX Volume 15
}

Abelew, T. A., Cuda, B. J., Koontz, J. E., Stell, J. C. and Johanson, M. A., Influence of Gender on Gastrocnemius Muscle Activity During the Stance Phase of Gait

15 (2012) 1250011

Acharya, K. K. V., see Pandey, V.

15 (2012) 1230001

Adams, B. D., see McCullough, M. B. A.

Adegoke, B. O. A., Akpan, G. A. and Mbada, C. E., Normative Values of Lower Back and Hamstring Flexibility for Nigerians Using the Modified Sit-and-Reach Test

15 (2012) 1250021

15 (2012) 1250015

Aihara, T., Toyone, T., Aoki, Y., Ozawa, T., Inoue, G., Hatakeyama, K. and Ouchi, J., Surgical Management of Degenerative Lumbar Spondylolisthesis: A Comparative Study of Outcomes Following Decompression with Fusion and Microendoscopic Decompression

15 (2012) 1250020

Akpan, G. A., see Adegoke, B. O. A.

15 (2012) 1250015

15 (2012) 1272004

15 (2012) 1250006

An, K.-N., see Kamineni, S.

15 (2012) 1250020

Artner, J., Cakir, B., Wernerus, D., Reichel, H. and Nelitz, M., Melorheostosis: Current Concepts in Diagnosis and Treatment. A Review of Literature (313 Cases)

15 (2012) 1230002

15 (2012) 1250012

15 (2012) 1250019

15 (2012) 1250010

15 (2012) 1272004

Britton, C., see Lee Spangler, M.

Broniecki, M., Esterman, A. and Grantham, H., Risk Factors for Back, Neck and Shoulder Musculoskeletal Injuries and Claims in Ambulance Officers

15 (2012) 1250009

15 (2012) 1230002

Crawford, R. J., Lynn, J. M., Malone, Q. and Price, R. I., Pain and Function One Year After Decompressive Disc Surgery: Cases Augmented with the DIAM Interspinous Implant Versus Those Receiving Microdiscectomy

15 (2012) 1250013

Crawford, R. J., Malone, Q. J. and Price, R. I., A Prospective Study of Patient-Reported Outcomes for Two Years After Lumbar Surgery Augmented with DIAM ${ }^{\circledR}$ Interspinous Implant

15 (2012) 1250018

Crispim, R., see Frazao, P. J. R.

15 (2012) 1250007

15 (2012) 1250011

Cuda, B. J., see Abelew, T. A.

15 (2012) 1250017

da Silva, L. C. L. C., see Moreira, R. C.

15 (2012) 1250019

Domire, Z. J., see Pauwels, L. L.

15 (2012) 1250017

do Valle de Zoppa, A. L., see Moreira, R. C.

15 (2012) 1250019

Dowling, B., see Pauwels, L. L.

15 (2012) 1250016 
Esterman, A., see Broniecki, M.

15 (2012) 1250009

Facó, L. L., see Frazao, P. J. R.

15 (2012) 1250007

Fonseca Pinto, A. C. B. C., see Frazao, P. J. R.

Frazao, P. J. R., Crispim, R., Pereira, C. A. M., Selim, M. B., Facó, L. L., Fonseca Pinto,

A. C. B. C., Hagen, S. C. F. and Zoppa, A. L. V., Correlation Study between Bone Mineral Density Determined by Radiographic Absorptiometry and Bone Resistance of Equine Third Metacarpal Bone Submitted to Biomechanical Testing

Frazão, P. J. R., see Moreira, R. C.

Grantham, H., see Broniecki, M.

Grosland, N. M., see McCullough, M. B. A.

Gupta, S., Sud, A., Sharma, D., Mehtani, A. and Parihar, A., Extra-Articular Osteochondroma in the Tibialis Anterior Tendon - Case Report

Hagen, S. C. F., see Frazao, P. J. R.

Hatakeyama, K., see Aihara, T.

Hoshi, M., Takada, J., Oebisu, N., Ieguchi, M. and Nakamura, H., Clinical Outcomes and Orthopedic Surgery for Bone Metastases from Hepatocellular Carcinoma

Ieguchi, M., see Hoshi, M.

Inoue, G., see Aihara, T.

Johanson, M. A., see Abelew, T. A.

Kamal, R. M., see Shanb, A.-S. A.

Kamineni, S., see Sasaki, K.

Kamineni, S., Wani, Z., Luo, Z.-P., Ruriko, Y. and An, K.-N., Chondrocyte Response to Tensile and Compressive Cyclic Loading Modalities

Karthikbabu, S., see Kumar C G, S.

Konagi, T., Takashi, O. and Kosuke, U., Open-Eyed One-Leg Standing Balance Time on Intact Leg of Hospitalized Elderly Leg Fracture Patients: Association with and Predictor of Locomotive Ability and Living Place After Hospital Discharge

Koontz, J. E., see Abelew, T. A.

Kosuke, U., see Konagi, T.

Kumar C G, S., Syed, N., Sirajudeen, M. S. and Karthikbabu, S., Position Sense Acuity Across Shoulder Rotational Range of Motion in Healthy Young Subjects

Kunamneni, A., see Sasaki, K.

Lee Spangler, M., Almusa, E. and Britton, C., A Persistent Median Artery and Bifid Median Nerve in the Setting of Carpal Tunnel Syndrome

Luo, Z.-P., see Kamineni, S.

Lynn, J. M., see Crawford, R. J.

Madigan, M. L., see Miller, E. M.

Malone, Q. J., see Crawford, R. J.

Malone, Q., see Crawford, R. J.

Martin, S. D., see Vercollone, C.

Mbada, C. E., see Adegoke, B. O. A.

McCullough, M. B. A., Adams, B. D. and Grosland, N. M., The Effect of Articular Surface Shape and Tendon Forces of Total Wrist Arthroplasty Systems: A Finite Element Study

Mehtani, A., see Gupta, S.

Mehtani, A., see Vijay, V.

Miller, E. M., Bazrgari, B., Nussbaum, M. A. and Madigan, M. L., Effects of Gender, Preload, and Trunk Angle on Intrinsic Trunk Stiffness

15 (2012) 1250007

15 (2012) 1250017

15 (2012) 1250009

15 (2012) 1250021

15 (2012) 1272003

15 (2012) 1250007

15 (2012) 1250020

15 (2012) 1250022

15 (2012) 1250022

15 (2012) 1250020

15 (2012) 1250011

15 (2012) 1250016

15 (2012) 1272006

15 (2012) 1250006

15 (2012) 1250014

15 (2012) 1250008

15 (2012) 1250011

15 (2012) 1250008

15 (2012) 1250014

15 (2012) 1272006

15 (2012) 1272004

15 (2012) 1250006

15 (2012) 1250013

15 (2012) 1250012

15 (2012) 1250018

15 (2012) 1250013

15 (2012) 1250010

15 (2012) 1250015

15 (2012) 1250021

15 (2012) 1272003

15 (2012) 1272005

15 (2012) 1250012 
Mohammadi, G., Risk Factors for the Prevalence of the Upper Limb and Neck Work-Related Musculoskeletal Disorders Among Poultry Slaughter Workers

15 (2012) 1250005

Moreira, R. C., Pereira, C. A. M., Frazão, P. J. R., Selim, M. B., da Silva, L. C. L. C. and do Valle de Zoppa, A. L., Evaluation of Bone Biomechanical Properties in Different Regions of the Equine Third Metacarpus

Nakamura, H., see Hoshi, M.

Nangia, A., see Vijay, V.

Nelitz, M., see Artner, J.

Nussbaum, M. A., see Miller, E. M.

Oebisu, N., see Hoshi, M.

Okafor, N., see Pauwels, L. L.

Ouchi, J., see Aihara, T.

Ozawa, T., see Aihara, T.

Pandey, V., van Laarhoven, S. N., Acharya, K. K. V., Rao, S. K. and Rao, S., Meniscal Abnormalities: Discoid Meniscus

Panwar, S., see Sureka, J.

Parihar, A., see Gupta, S.

Pauwels, L. L., Dowling, B., Okafor, N., Breighner, R. and Domire, Z. J., Calorie Restriction as a Means to Control Skeletal Muscle Stiffness in Aged Rats

Pereira, C. A. M., see Frazao, P. J. R.

Pereira, C. A. M., see Moreira, R. C.

Price, R. I., see Crawford, R. J.

Price, R. I., see Crawford, R. J.

Rao, S. K., see Pandey, V.

Rao, S., see Pandey, V.

Rashidifard, C., see Vercollone, C.

Reichel, H., see Artner, J.

Ruriko, Y., see Kamineni, S.

Sasaki, K., Kunamneni, A., Sinai, A. and Kamineni, S., Analysis of Collagen Profile and Orientation in a Chronic Ruptured Extensor Pollicis Longus Tendon

Selim, M. B., see Frazao, P. J. R.

Selim, M. B., see Moreira, R. C.

Shanb, A.-S. A., Youssef, E. F., El-Barkouky, M. G., Kamal, R. M. and Tawfick, A. M., The Effect of Magnetic Therapy and Active Exercise on Bone Mineral Density in Elderly Women with Osteoporosis

Sharma, D., see Gupta, S.

Sinai, A., see Sasaki, K.

Sirajudeen, M. S., see Kumar C G, S.

Stell, J. C., see Abelew, T. A.

Sud, A., see Gupta, S.

Sud, A., see Vijay, V.

Sureka, J. and Panwar, S., MR Imaging of Suprascapular Neuropathies

Syed, N., see Kumar C G, S.

Takada, J., see Hoshi, M.

Takashi, O., see Konagi, T.

Tawfick, A. M., see Shanb, A.-S. A.

Toyone, T., see Aihara, T.

van Laarhoven, S. N., see Pandey, V.

15 (2012) 1250017

15 (2012) 1250022

15 (2012) 1272005

15 (2012) 1230002

15 (2012) 1250012

15 (2012) 1250022

15 (2012) 1250019

15 (2012) 1250020

15 (2012) 1250020

15 (2012) 1230001

15 (2012) 1230003

15 (2012) 1272003

15 (2012) 1250019

15 (2012) 1250007

15 (2012) 1250017

15 (2012) 1250013

15 (2012) 1250018

15 (2012) 1230001

15 (2012) 1230001

15 (2012) 1250010

15 (2012) 1230002

15 (2012) 1250006

15 (2012) 1272006

15 (2012) 1250007

15 (2012) 1250017

15 (2012) 1250016

15 (2012) 1272003

15 (2012) 1272006

15 (2012) 1250014

15 (2012) 1250011

15 (2012) 1272003

15 (2012) 1272005

15 (2012) 1230003

15 (2012) 1250014

15 (2012) 1250022

15 (2012) 1250008

15 (2012) 1250016

15 (2012) 1250020

15 (2012) 1230001 
Vercollone, C., Rashidifard, C., Zan, S., Martin, S. D. and Brezinski, M. E., New Technological Approach to Study Rotator Cuff Pathology

15 (2012) 1250010

Vijay, V., Sud, A., Mehtani, A. and Nangia, A., Expansile Lytic Lesion in Medial End Clavicle in an Adolescent Girl: A Case Report

15 (2012) 1272005

Wani, Z., see Kamineni, S.

15 (2012) 1250006

Wernerus, D., see Artner, J.

15 (2012) 1230002

Youssef, E. F., see Shanb, A.-S. A.

15 (2012) 1250016

Zan, S., see Vercollone, C.

15 (2012) 1250010

Zoppa, A. L. V., see Frazao, P. J. R.

15 (2012) 1250007 